\title{
Reflets
}

Revue d'intervention sociale et communautaire

\section{Sensibilisation, structuration et consolidation en santé en français : 10 ans d'action}

\section{Société Santé en français}

Volume 20, numéro 2, automne 2014

URI : https://id.erudit.org/iderudit/1027590ar

DOI : https://doi.org/10.7202/1027590ar

Aller au sommaire du numéro

Éditeur(s)

Reflets, Revue d'intervention sociale et communautaire

ISSN

1203-4576 (imprimé)

1712-8498 (numérique)

Découvrir la revue

Citer cet article

Société Santé en français (2014). Sensibilisation, structuration et consolidation en santé en français : 10 ans d'action. Reflets, 20(2), 166-177.

https://doi.org/10.7202/1027590ar d'utilisation que vous pouvez consulter en ligne. 


\section{Sensibilisation, structuration et consolidation en santé en français : 10 ans d'action}

$\sqrt{2}$ Société Santé en français

Fondée en 2002, la Société Santé en français est un organisme pancanadien, à but non lucratif, qui a pour mandat d'assurer un meilleur accès à des services ou à des programmes de santé et de services sociaux de qualité en français, en vue d'améliorer l'état de santé de l'ensemble des communautés francophones et acadiennes vivant en situation minoritaire. La Société se compose d'un secrétariat national et de 16 réseaux de santé en français situés dans toutes les provinces et territoires, sauf au Québec.

Les réseaux de santé en français sont des entités autonomes " au cœur de l'action ». Euvrant sur le terrain, ils comprennent bien les besoins et les priorités de leurs communautés et visent à trouver des moyens d'améliorer l'accès aux services de santé dans leurs milieux. Les réseaux de santé «sont perçus comme étant des centres de liaison pour la gestion des préoccupations en matière de santé des [communautés de langue officielle en situation minoritaire] dans bon nombre de provinces et de territoires " (Santé Canada, 2013). Plusieurs d'entre eux participent ainsi aux processus de planification de leur province ou territoire et sont parties prenantes dans la mise en œuvre des stratégies d'action qui en découlent. 
Cependant, comme l'environnement juridique ou politique dans lequel ils agissent varie d'une province ou d'un territoire à l'autre, les réseaux doivent adapter leurs stratégies et actions à leurs réalités respectives. Depuis leur création, plusieurs réseaux ont obtenu une reconnaissance officielle ou sont appelés à jouer un rôle formel; ils sont interpellés par leurs décideurs politiques ou leurs systèmes de santé comme planificateurs, experts-conseils ou comme représentants de leurs communautés. Ces réseaux ont la possibilité de recommander des actions structurantes visant à augmenter l'accès à des services professionnels ou à en accroître l'offre. D'autre part, des réseaux travaillent aussi à sensibiliser les instances dirigeantes à l'importance de l'accès pour leurs communautés à des services de santé et à des services sociaux en français. À cet effet, il est possible de constater chaque année des avancées et des progrès significatifs, grâce à l'approche de réseautage choisie.

La Société Santé en français et les réseaux de santé du pays $œ u v r e n t$ sur plusieurs plans. En organisation de services, par exemple, l'outillage et le soutien de professionnelles ou de professionnels favorisent l'offre active et aident à réduire les barrières à l'accès de services de santé et de services sociaux de qualité en français. Les réalisations du Mouvement santé en français sont intimement liées à l'approche caractéristique de la Société Santé en français et des réseaux, soit celle du réseautage. Dans le présent article, nous exposons brièvement cette approche et nous présentons certaines réalisations de la Société Santé en français et de ses réseaux, ainsi que les défis et enjeux principaux associés à l'offre de services de santé et de services sociaux en français, de même que les grandes lignes du plan directeur quinquennal de la Société.

\section{Le réseautage en santé}

Depuis ses débuts, la Société Santé en français a adopté le modèle de réseautage de l'Organisation mondiale de la santé (OMS), Vers l'unité pour la santé. La force de l'approche de la Société Santé en 
français repose sur le choix stratégique d'associer les principales parties concernées par les systèmes de santé à nos actions. Au cœur de nos activités se trouvent donc les gouvernements provinciaux et territoriaux ou les autorités régionales, les établissements ou gestionnaires du système de santé, les professionnelles ou professionnels de la santé et des services sociaux, les représentants des communautés et les institutions de formation. Sans la participation de ces partenaires-clés, l'obtention de changements durables dans les systèmes de santé et de services sociaux serait impossible.

Les partenaires-clés de la Société Santé en français agissent ainsi en tant qu'experts sur l'accessibilité aux services de santé en français et s'assurent que les activités de la Société Santé en français s'intègrent dans les planifications et programmations gouvernementales. Grâce à ce modèle, les besoins réels des francophones et des Acadiens vivant en situation minoritaire sont toujours au centre des préoccupations.

Pour la Société Santé en français, le réseautage comprend toute une série d'activités qui visent à :

- pallier les ressources limitées dans ces communautés et combattre la méconnaissance de la situation des francophones en matière de santé et de services sociaux;

- faciliter la navigation de ces communautés dans les systèmes de santé existants;

- accroître leur influence dans les décisions prises par les institutions et les établissements;

- contrer la fragmentation des interventions et l'isolement des professionnelles ou professionnels de la santé et des services sociaux;

- responsabiliser chacun des acteurs dans leur prise en charge du défi d'un meilleur accès aux services de santé en français.

Le réseautage s'avère être un excellent moyen d'établir des relations porteuses et durables entre les partenaires de la santé. La connexion et la collaboration engendreront une meilleure évaluation des besoins des communautés francophones et acadiennes en situation minoritaire et par la suite, l'adoption de solutions adaptées et efficaces aux besoins identifiés. 


\section{La santé en français progresse}

Un nombre grandissant de personnes et de milieux ont bénéficié des avancées significatives réalisées dans toutes les régions du pays en matière de santé et de services sociaux en français. Ces réalisations de la Société Santé en français et des réseaux, en collaboration avec les partenaires, ont permis d'améliorer l'accès à des services de santé en français. Parmi les réalisations des dix dernières années, notons les suivantes:

- Entre 2010 et 2013, 18 projets d'intégration des services de santé et 68 initiatives santé menées dans l'ensemble des provinces et territoires en santé mentale, petite enfance, enfants et jeunes, aînés, en accès global et en promotion de la santé. Dans le cadre de ces initiatives, ce sont plus de 350 outils et mécanismes d'information qui ont été développés par les réseaux et la Société Santé en français;

- Une des réalisations structurantes des dernières années est la définition des Orientations en santé mentale en français, en collaboration avec la Commission de la santé mentale du Canada;

- Plusieurs réseaux participent maintenant de façon active aux processus de planification de leur province ou territoire et sont parties prenantes dans la mise en œuvre des stratégies d'action qui en découlent;

- Plus de 250 organismes ont participé à des projets de promotion de la santé au sein des communautés francophones et acadiennes. Dans les Territoires du Nord-Ouest, par exemple, l'accent a été mis sur la prévention des situations d'urgence par la formation des étudiantes et des étudiants et du personnel enseignant francophone à tous les niveaux jusqu'à la $12^{\mathrm{e}}$ année. Près de la moitié des publications rédigées dans le cadre de projets parrainés par les réseaux traitent ainsi de la promotion de la santé;

- De nombreux programmes issus des systèmes de santé 
provinciaux et territoriaux ont été traduits et adaptés par les réseaux et leurs partenaires pour les CAFSM et diffusés à d'autres régions. Citons les programmes Mieux vivre avec une maladie chronique de la Saskatchewan et Grandir en santé de la Colombie-Britannique, pour n'en nommer que deux;

- Plus de 450 intervenantes et intervenants de la santé ont participé au colloque national de la Société Santé en français, tenu à Ottawa en novembre 2012. Ce colloque a accueilli cinq ministres fédéraux, provinciaux ou territoriaux, ainsi que le premier ministre manitobain.

Ce témoignage d'un expert dans le domaine illustre la portée et les retombées positives de cette dernière activité :

À l'observateur extérieur que je suis depuis le début de la création du mouvement santé en français, trois traits fondamentaux se sont révélés lors du dernier Rendez-vous 2012. D'abord, la motivation de ses membres dont la ferveur initiale s'est maintenue, voire, amplifiée : elle agit comme une formidable énergie pour surmonter les défis et garder le cap sur les engagements. Ensuite, l'épanouissement des programmes au sein des réseaux qui se sont consolidés, diversifiés et dont la gouvernance s'est améliorée, laissant présager une pérennité des actions entreprises. Enfin [il existe] une reconnaissance éblouissante par les autorités politiques, sanitaires et académiques de la qualité des accomplissements. (Boelen, 2012, p.1)

\section{La langue comme enjeu incontournable en santé}

La langue de communication entre la professionnelle ou le professionnel qui prodigue un service de santé et la personne qui le reçoit est un facteur important quant à l'efficience dudit service et la sécurité du patient qui en découle. Une communication 
claire est essentielle à l'établissement d'un diagnostic juste et à l'adhésion de la personne à son traitement.Autrement, les barrières linguistiques entrânent des conséquences pour le système de santé et la personne elle-même, et constituent un facteur d'inefficacité du système de santé et d'imputabilité.

L'accessibilité à des soins de santé en langue française ne constitue pas un caprice, un souhait, mais une nécessité justifiée, étudiée, mesurée scientifiquement. En effet, les recherches et organisations qui se penchent sur le lien entre la maitrise de la langue, les soins de santé et l'état des personnes sont de plus en plus nombreuses. Plusieurs experts reconnaissent et ont fait la démonstration de l'importance de la langue dans l'efficacité des services. (Bowen, 2001; Green, 2007; Bouchard, 2009)

$\mathrm{La}$ recherche indique que les problèmes de communication ont un impact négatif, entre autres, sur l'accès et la fidélité aux traitements, sur la participation à des mesures de prévention, sur la capacité d'obtenir un véritable consentement et sur la qualité des soins (Fortin, 2012).

Or, la prise en compte de la langue dans l'organisation des services de santé et la reconnaissance du droit des francophones à obtenir des services de santé dans leur langue ne sont pas encore des réalités dans les systèmes de santé canadiens.

\section{Le rôle des ressources humaines}

La disponibilité de ressources humaines en nombre suffisant est un défi majeur pour tous les systèmes de santé. Dans certaines régions du pays, le bassin de ressources humaines ayant les compétences linguistiques est souvent limité et cet enjeu ira en s'accentuant au cours des prochaines années. Il faudra trouver d'autres approches de développement des ressources humaines capables d'offrir les services en français à partir de l'interne. En effet, il faudra convaincre les fournisseurs de services à mieux connaître leur propre capacité en s'assurant de faire la collecte de la variable linguistique auprès de leur personnel. 
Nous savons que les employés bilingues cherchent des milieux de travail où ils peuvent utiliser le français dans un climat où ils se sentent appréciés et autorisés à le faire. Il faudra également favoriser la création de tels environnements. Le recrutement en contexte minoritaire nécessite une approche adaptée, une attitude d'attraction et une approche d'intégration.

Les réalisations du Consortium national de formation en santé en français ont contribué de façon importante à la formation d'une main-d'œuvre qualifiée en français. Par ailleurs, la Société Santé en français croit qu'il faut aussi mettre l'accent sur les intervenantes et intervenants en santé déjà sur le terrain, qui parlent le français, mais qui ne sont pas nécessairement reconnus, valorisés ou outillés. Plusieurs d'entre eux exercent dans des milieux anglophones et constituent également un bassin de ressources humaines non exploité pour les services en français.

\section{Destination santé 2018}

En novembre 2012, la Société Santé en français s'est dotée d'un nouveau plan directeur quinquennal intitulé Destination Santé 2018 , lequel élabore un modèle d'interventions coordonnées favorisant un accès équitable et efficace à des services de santé de qualité et linguistiquement adaptés aux besoins des communautés francophones et acadiennes vivant en situation minoritaire.

Les quatre champs d'action prioritaires identifiés résultent d'une planification stratégique menée aux niveaux national, provincial et territorial. Ils reflètent les priorités qui se dégagent des consultations et réflexions conduites auprès des communautés et des partenaires dans les réseaux Santé en français. Elles se résument comme suit :

\section{L'organisation et l'adaptation des services pour une meilleure accessibilité}

La Société Santé en français et les réseaux contribuent à ce que l'offre de services de santé en français soit améliorée de façon importante grâce à l'intégration des besoins des francophones 
dans la mise en œuvre de services de santé de qualité, en français, tout au long du continuum des services de santé. Les systèmes de santé partout au pays favorisent de plus en plus la mise en place de services intégrés et de continuum de soins afin de mieux servir leur population, tout en générant des efficacités d'échelle et de coûts.

\section{La concertation, la valorisation et l'outillage des ressources humaines pour la qualité et la sécurité des services en français}

Les ressources humaines sont un facteur clé de l'accès à des soins et des services de santé de qualité en français. Une prestation sécuritaire de services exige en effet que les dispensateurs puissent comprendre le besoin du client et lui transmettre l'information essentielle à un choix éclairé et à un traitement efficace. Les activités de ce volet s'inscrivent donc en continuité avec celle du volet Réseautage en santé. Elles sont aussi intégrées et complémentaires avec les trois autres champs d'action visant l'amélioration de l'accès aux services de santé en français et le maintien en poste de professionnelles ou de professionnels qui puissent offrir des services en français.

Assurer une offre de services en français adéquate nécessite des efforts importants et supplémentaires à ceux habituellement consentis par les autorités de santé ou par les dirigeants d'établissements de santé.Appuyés par leur province ou territoire, ou par les autorités régionales, certains réseaux jouent déjà un rôle important à cet égard; selon leur contexte, d'autres ont besoin d'un plus grand appui et d'approches différentes et adaptées pour rejoindre les professionnelles et les professionnels francophones et les engager à offrir des services en français.

Par ailleurs, les partenaires au sein des gouvernements provinciaux ou territoriaux, les organismes et les associations professionnelles du domaine de la santé sont prêts à travailler avec les réseaux et la Société Santé en français afin de poursuivre les avancées en matière de ressources humaines pour la santé en français. De ce fait, il est essentiel non seulement 
d'avoir des ressources humaines outillées, mais aussi d'instaurer des politiques qui placent les compétences linguistiques et culturelles au cœur du processus de qualité de services.

\section{L'action sur les déterminants de la santé pour accroître le mieux-être en français}

La disponibilité de services de santé constitue un des neuf déterminants de la santé. En d'autres mots, plus il y a de services préventifs et de soins primaires disponibles, meilleur est l'état de santé. Les efforts de la Société Santé en français, comme ceux des réseaux et des partenaires, portent sur l'amélioration de l'accès des communautés francophones et acadiennes vivant en situation minoritaire à une gamme de services de santé de qualité en français et sur l'appui à apporter à ces dernières dans la prise en charge de leur santé et de leur mieux-être au chapitre des déterminants de la santé.Ainsi, non seulement veut-on promulguer la disponibilité de soins, mais aussi instiguer la promotion de la santé et du mieux-être pour améliorer l'état de santé de ces communautés.

Pour ce faire, les communautés et les individus doivent prendre en charge leur santé; ils doivent contribuer à leur mieux-être en reconnaissant les facteurs déterminant leur état de santé et, au besoin, prendre des décisions et apporter des changements nécessaires. Le but de la proposition Promotion de la santé est de créer un environnement qui engendrera cette prise en charge.

\section{Le transfert des connaissances fondé sur la recherche et l'évaluation pour atteindre une qualité optimale de services}

Ce champ d'action prioritaire vise à évaluer la mise en œuvre de la programmation de la Société Santé en français et de ses réseaux et son impact sur l'amélioration de l'accès à des services de santé de qualité en français dans les communautés de langues officielles en situation minoritaire. La Société veut se doter de moyens pour mesurer et maximiser l'impact de ses actions et de celles des réseaux sur les communautés francophones et acadiennes vivant en situation minoritaire. Pour ce faire, elle adoptera une méthode de suivis et d'évaluation plus 
systématique du rendement et s'assurera que les actions donnent les résultats souhaités et contribuent aux objectifs plus globaux. Enfin, la Société Santé en français mettra sur pied un Réseau de transfert de connaissances.

D'ici 2018, pour chaque champ d'action prioritaire, la Société Santé en français et les réseaux se sont donné des "dossiers leviers " qui constituent leurs grandes priorités d'action. Grâce à ces dossiers prioritaires, la Société Santé en français compte réaliser des transformations significatives dans l'accès aux services de santé par des actions structurantes et bien coordonnées. Ces priorités d'action consistent à :

Mobiliser et appuyer les partenaires dans la mise en place de conditions gagnantes de l'offre active

D'ici 2018, l'offre active de services de santé en français tout au long du continuum de services doit être significativement augmentée. En collaboration avec le Consortium national de formation en santé en français, la Société Santé en français et les réseaux vont travailler avec leur système de santé et services sociaux pour assurer la mise en œuvre d'initiatives d'offre active partout au pays.

Amorcer la mise en place des Orientations en santé mentale

D'ici 2018, l'offre de service aux populations francophones vulnérables sera améliorée par la mise en œuvre des Orientations en santé mentale en français, développées au cours de la période 2008-2013.

Soutenir le développement et le déploiement d'initiatives structurantes de type Communautés et écoles en santé

Selon les particularités provinciales, territoriales ou locales, la Société Santé en français et les réseaux verront à organiser des services de promotion de la santé et de mieux-être en français dans une convergence nationale, régionale et locale; l'objectif est la création de milieux propices à la santé et au mieux-être en français et l'augmentation de l'accès aux programmes en français de prévention des maladies, de promotion de la santé et de mieux-être. 
Mobiliser et concerter les partenaires clés concernés par les ressources humaines pouvant offrir des services en français

Cela se fera en favorisant la création de communautés d'accueil pour les professionnelles ou professionnels francophones désirant s'installer dans les communautés éloignées; et par le recrutement et l'accueil des professionnelles ou des professionnels de la santé qui parlent français dans les communautés, les établissements et organisations de santé.

Engager les acteurs-clés des systèmes de santé pour qu'ils agissent sur le développement de normes de services de santé culturellement et linguistiquement adaptés

Les autorités, les fournisseurs, les professionnelles ou professionnels et les organismes de réglementation et les milieux de formation reconnaissent l'importance de l'accessibilité linguistique en français comme un élément clé de qualité, de sécurité et d'efficience.

Promouvoir l'intégration de la variable linguistique dans les collectes de données en santé, tant pour les clientèles que pour les professionnelles ou professionnels

Cette inclusion de la variable linguistique permettra d'identifier les besoins des communautés francophones et acadiennes en matière de santé et de services de santé en français afin qu'ils soient pris en compte par les systèmes de santé et autres partenaires et pourvoyeurs de services.

Développer un mécanisme et des outils dans le but de favoriser le transfert de connaissances et de bonnes pratiques

Ce dossier se penchera sur la création d'un réseau de transfert de connaissances et le développement d'une banque de modèles, d'outils et de bonnes pratiques visant l'amélioration de l'accès aux services de santé en français novateurs qui ont fait leurs preuves dans un contexte déterminé. 


\section{Conclusion}

Forts de dix années d'expérience et de réalisations, la Société Santé en français et les réseaux santé en français utiliseront cette expertise pour atteindre la vision décrite dans Destination Santé 2018. Ainsi, avec l'appui financier de Santé Canada, dans le cadre de la Feuille de route sur les langues officielles, et le travail de réseautage avec tous ses partenaires, la Société Santé en français et les réseaux visent à améliorer l'accès équitable et efficace, à augmenter le nombre de francophones desservis dans leur langue, de partenariats, d'initiatives et de programmes intégrés dans le système. Ils visent également à augmenter le nombre de communautés touchées par les initiatives des réseaux et de la Société Santé en français afin que l'état de santé des francophones s'améliore partout au pays.

\section{Bibliographie}

BOELEN, Charles (2012). Allocution de fermeture du Rendez-vous de la santé en français 2012, [Rapport d'une allocution au $6^{\text {e }}$ Rendez-vous de la santé en français], Ottawa.

BOUCHARD, Louise (2009). Les déterminants de la santé en situation linguistique minoritaire, [Colloque scientifique :La santé des communautés de langue officielle en situation minoritaire du Canada], BACLO, Santé Canada, Ottawa, 2009.

BOWEN, Sarah (2001). Barrières linguistiques dans l'accès aux soins de santé, [Rapport de recherche], Canada, Santé Canada, $141 \mathrm{p}$.

FORTIN, Jacques (2012). Regard sur les populations [Rapport d'une allocution au $6^{\mathrm{e}}$ Rendez-vous de la santé en français], Ottawa.

GREEN, Alexander R. (2007). The impact of language barrier on health care, [Rapport d'une allocution au $3^{\text {e }}$ Rendez-vous de la santé en français], Ottawa, $52 \mathrm{p}$.

SANTÉ CANADA (2013). Évaluation du Programme de contribution pour les langues officielles en santé 2008-2012, [Rapport d'évaluation], Ottawa, 66 p. 